Article

\title{
Analysis of Generalized Multistep Collocation Solutions for Oscillatory Volterra Integral Equations
}

\author{
Hao Chen, Ling Liu and Junjie Ma * \\ School of Mathematics and Statistics, Guizhou University, Guiyang 550025, China; sdch0807@163.com (H.C.); \\ lingliu95@126.com (L.L.) \\ * Correspondence: jjma@gzu.edu.cn
}

Received: 9 October 2020; Accepted: 4 November 2020; Published: 10 November 2020

check for updates

\begin{abstract}
In this work, we introduce a class of generalized multistep collocation methods for solving oscillatory Volterra integral equations, and study two kinds of convergence analysis. The error estimate with respect to the stepsize is given based on the interpolation remainder, and the nonclassical convergence analysis with respect to oscillation is developed by investigating the asymptotic property of highly oscillatory integrals. Besides, the linear stability is analyzed with the help of generalized Schur polynomials. Several numerical tests are given to show that the numerical results coincide with our theoretical estimates.
\end{abstract}

Keywords: collocation; volterra integral equation; highly oscillatory; convergence

\section{Introduction}

In many practical problems, such as epidemic diffusion, population dynamics and reaction processes, one may usually come across a class of Volterra integral equations (VIEs) (see [1] and references therein). Noting that most VIEs cannot be solved in closed forms, many researchers have made contributions to the numerical approaches to VIEs.

Particularly, the study of numerical solutions to VIEs with highly oscillatory Fourier or Bessel kernels has attracted much attention during the past decade. In [2], Xiang and Brunner first investigated Filon collocation approximations to highly oscillatory VIEs by employing the asymptotic property of oscillatory integrals. They found that errors of Filon collocation solutions decayed fast as the frequency increased. The third author presented an optimal convergence order for the direct Filon collocation solution to the first kind of oscillatory VIE arising in acoustic scattering in [3]. The convergence behavior of such kinds of numerical approaches was able to be revealed with the help of the detailed study of the remainder for the error function. Besides, it is noted that numerical analysis with respect to the frequency, which is usually done by solving error equations and extending van der Corput lemma (see [4] p. 333), is able to detect the ability of the numerical method to solve highly oscillatory VIEs. With these techniques in mind, several authors made great contributions to numerical solutions to highly oscillatory VIEs. For example, Galerkin and collocation solutions for VIEs with highly oscillatory trigonometric kernels were investigated in $[5,6]$, highly oscillatory VIEs with weakly singular kernels were studied in [7], the Hermite-type Filon collocation method was presented in [8], and Clenshaw-Curtis-Filon qudrature for Cauchy singular integral equations was investigated in [9].

In this work, we consider the numerical computation of the following second-kind oscillatory VIE:

$$
u(t)=f(t)+\int_{0}^{t} K(t, s) \mathrm{e}^{\mathrm{i} \omega g(t, s)} u(s) d s, t \in[0, T],
$$

where $K(t, s), g(t, s)$ and $f(t)$ are sufficiently smooth, $u(t)$ is unknown, and $\omega$ denotes the oscillation parameter. When $\omega=0$, Equation (1) reduces to the classical VIEs. In the case of $\omega \gg 1$, the kernel 
in Equation (1) is highly oscillatory, and special quadrature rules should be employed in practical computation.

In the remaining part, we are restricted to the following problems. In the forthcoming section, we first develop a class of generalized multistep collocation methods $\left(G M C_{k_{1}, k_{2}} M\right)$ for Equation (1) with non-oscillatory kernels, that is, $\omega=0$. Then, classical convergence analysis and linear stability analysis are implemented. In the third section, we study the numerical solution to VIE (1) when the kernel changes rapidly, that is, $\omega \gg 1$, and present the frequency-explicit convergence analysis. Some concluding remarks are given in Section 4.

\section{2. $\mathrm{GMC}_{\mathrm{k}_{1}, \mathrm{k}_{2}} \mathrm{M}$ in the Case of $\omega=0$}

Frequently-used approaches for VIEs include collocation methods [10], the spectral collocation method [11,12], the spectral Galerkin method [13,14], the Nyström method [15,16], and so on. Among these numerical formulae, the collocation-based approach is one of the most important tools. In general, the collocation solution is obtained by making the polynomial or piecewise polynomial satisfy the collocation equation. For one-step collocation methods, one can find detailed analysis in [10]. To increase the convergence rate without adding collocation points, Conte and Paternoster studied multistep collocation solutions with the help of employing approximations to numerical solutions in computed steps in [17]. However, multistep methods usually tend to be unstable. Fazeli et al. further investigated the stability of multistep collocation methods in [18], and found some super implicit collocation solutions with wide stability regions. On the other hand, inspired by the study of boundary value methodology for solving ODE (see [19]), several authors made contributions to boundary value solutions to Volterra functional equations [20-22]. Based on interpolation outside the current subinterval and approximated end values, the third author and Xiang devised CBVM for second-kind VIEs in [22]. Furthermore, the third author extended said kind of methodology to VIEs with weakly singular kernels by employing the fractional polynomial interplant in [23], and the block CBVM for the first-kind VIE was investigated in [24]. In this section we first investigate the construction of $G M C_{k_{1}, k_{2}} M$ with the help of local polynomial interpolation. Then, the convergence and linear stability analysis of $G M C_{k_{1}, k_{2}} M$ are considered.

\subsection{Discretization of VIE}

Let the interval $[0, T]$ be divided uniformly, that is,

$$
X_{h}=\left\{t_{j}: t_{j}=j h, j=0,1, \cdots, N=T / h\right\} .
$$

Then define local basic functions

$$
\phi_{j}^{k_{1}, k_{2}}(s)=\prod_{i=-k_{1}, i \neq j}^{k_{2}+1} \frac{s-i}{j-i}, j=-k_{1}, \cdots, k_{2}+1,
$$

For the first $k_{1}$ subintervals, that is, for any $t \in\left[t_{0}, t_{k_{1}}\right]$, the collocation polynomial is represented by

$$
u_{h}\left(t_{k_{1}}+s h\right)=\sum_{i=-k_{1}}^{k_{2}+1} y_{k_{1}+i} \phi_{i}^{k_{1}, k_{2}}(s), s \in\left(-k_{1}, 0\right]
$$

For $k_{1} \leq n \leq N-k_{2}-1, u_{h}(t)$ over the interval $\left[t_{n}, t_{n+1}\right]$ is rewritten as

$$
u_{h}\left(t_{n}+s h\right)=\sum_{i=-k_{1}}^{k_{2}+1} y_{n+i} \phi_{i}^{k_{1}, k_{2}}(s), s \in(0,1]
$$


In the last subinterval $\left[t_{N-k_{2}}, t_{N}\right]$, we rewrite $u_{h}(t)$ as

$$
u_{h}\left(t_{N-k_{2}-1}+s h\right)=\sum_{i=-k_{1}}^{k_{2}+1} y_{N-k_{2}-1+i} \phi_{i}^{k_{1}, k_{2}}(s), s \in\left(1, k_{2}+1\right] .
$$

Finally, the collocation equation follows:

$$
u_{h}\left(t_{n}\right)=f\left(t_{n}\right)+\int_{0}^{t_{n}} K\left(t_{n}, s\right) u_{h}(s) d s, t_{n} \in X_{h}
$$

A direct calculation leads to

$$
y_{n}-f\left(t_{n}\right)= \begin{cases}h \int_{-k_{1}}^{n-k_{1}} K\left(t_{n}, t_{k_{1}}+s h\right)\left(\sum_{i=-k_{1}}^{k_{2}+1} y_{k_{1}+i} \phi_{i}^{k_{1}, k_{2}}(s)\right) d s, & n=1, \cdots, k_{1}, \\ h \int_{-k_{1}}^{0} K\left(t_{n}, t_{k_{1}}+s h\right)\left(\sum_{i=-k_{1}}^{k_{2}+1} y_{k_{1}+i} \phi_{i}^{k_{1}, k_{2}}(s)\right) d s & \\ +h \sum_{j=k_{1}+1}^{n} \int_{0}^{1} K\left(t_{n}, t_{j-1}+s h\right)\left(\sum_{i=-k_{1}}^{k_{2}+1} y_{j-1+i} \phi_{i}^{k_{1}, k_{2}}(s)\right) d s, & n=k_{1}+1, \cdots, N-k_{2}, \\ h \int_{-k_{1}}^{0} K\left(t_{n}, t_{k_{1}}+s h s h\right)\left(\sum_{i=-k_{1}}^{k_{2}+1} y_{k_{1}+i} \phi_{i}^{k_{1}, k_{2}}(s)\right) d s & \\ +h \sum_{j=k_{1}+1}^{N-k_{2}} \int_{0}^{1} K\left(t_{n}, t_{j-1}+s h\right)\left(\sum_{i=-k_{1}}^{k_{2}+1} y_{j+i-1} \phi_{i}^{k_{1}, k_{2}}(s)\right) d s & \\ +h \int_{1}^{n-N+k_{2}+1} K\left(t_{n}, t_{N-k_{2}-1}+s h\right) \sum_{i=-k_{1}}^{k_{2}+1} y_{N-k_{2}-1+i} \phi_{i}^{k_{1}, k_{2}}(s) d s, \quad n=N-k_{2}+1, \cdots, N .\end{cases}
$$

Denoting

$$
\operatorname{MOM}_{a, c, i}^{b, d}=\int_{a}^{b} K\left(t_{c}, t_{d}+s h\right) \phi_{i}^{k_{1}, k_{2}}(s) d s,
$$

we have for $k=-k_{1}, \cdots, k_{2}+1$,

$$
\begin{gathered}
A_{k}^{\text {initial }}=\left(a_{i, j}^{(k)}\right)= \begin{cases}\operatorname{MOM}_{-k_{1}, n, j-k_{1}-1}^{0, k_{1}} & i \leq N, j=k+k_{1}+1, \\
0, & \text { others, }\end{cases} \\
A_{k}^{\text {main }}=\left(b_{i, j}^{(k)}\right)= \begin{cases}\operatorname{MoM}_{0, n, k}^{1, j-1}, & k_{1}<i \leq N, i+k \leq j \leq i+k+\min \left\{0, n-N+k_{2}+1\right\}, \\
0, & \text { others, }\end{cases} \\
A_{k}^{\text {end }}=\left(c_{i, j}^{(k)}\right)= \begin{cases}\operatorname{MoM}_{i, n, j-N+k_{2}}^{n-N+k_{2}+1, N-k_{2}-1}, & i>N-k_{2}, j=N-k_{2}+k, \\
0, & \text { others. }\end{cases}
\end{gathered}
$$

Now we are able to rewrite Equation (6) in the closed form:

$$
(\mathbf{I}-h \mathbf{A}(1: N, 2: N+1)) \mathbf{Y}=\mathbf{F}+h y_{0} \mathbf{A}(1: N, 1),
$$

where $\mathbf{I}$ denotes the identity matrix, $\mathbf{A}=\sum_{k=-k_{1}}^{k_{2}+1} A_{k}^{\text {initial }}+\sum_{k=-k_{1}}^{k_{2}+1} A_{k}^{\text {main }}+\sum_{k=-k_{1}}^{k_{2}+1} A_{k}^{\text {end }}, \mathbf{Y}=$ $\left[y_{1}, y_{2}, \cdots, y_{N}\right]^{T}$, and $\mathbf{F}=\left[f\left(t_{1}\right), f\left(t_{2}\right), \cdots, f\left(t_{N}\right)\right]^{T}$. By employing proper numerical integration approaches such as Clenshaw-Curtis quadrature and applying iterative solvers to Equation (9), we are able to obtain the collocation solution at the grid. 


\subsection{Convergence Analysis with Respect to Stepsize}

Now we turn to studying the convergence behavior of the piecewise collocation polynomial computed by Equation (9). Firstly, we revisit some helpful results from approximation theory.

Lemma 1 ([10] p. 43). Consider the following assumption.

- Defining abscissa $a \leq \xi_{1}<\ldots<\xi_{m} \leq b$, we obtain the error between $f(x)$ and the Lagrange interpolation polynomial of degree $m-1$ with respect to the given points $\left\{\xi_{j}\right\}$.

$$
\varepsilon_{m}(f ; x)=f(x)-\sum_{j=1}^{m} L_{j}(x) f\left(\xi_{j}\right), x \in[a, b]
$$

where $L_{j}(x)$ denotes Lagrange basis.

- Letting $1 \leq d \leq m$, we suppose $f(x)$ belongs to the space $C^{d}[a, b]$.

Then we can represent the error function $\varepsilon_{m}(f ; x)$ as follows.

$$
\varepsilon_{m}(f ; x)=\int_{a}^{b} \kappa_{d}(x, t) f^{(d)}(t) d t, x \in[a, b] .
$$

Here the kernel function $\kappa_{d}(x, t)$ can be obtained by

$$
\kappa_{d}(x, t):=\frac{1}{(d-1) !}\left\{(x-t)_{+}^{d-1}-\sum_{j=1}^{m} L_{j}(x)\left(\xi_{j}-t\right)_{+}^{d-1}\right\},
$$

and

$$
(x-t)_{+}^{p}:=\left\{\begin{array}{l}
0, x<t \\
(x-t)^{p}, x \geq t .
\end{array}\right.
$$

Lemma 2 ([10] p. 81). Suppose that there a sequence $\left\{k_{i}\right\}$ with $k_{i} \geq 0$ and another sequence $\left\{\epsilon_{i}\right\}$ with $\epsilon_{0} \leq \rho_{0}$. Moreover, $\left\{k_{i}\right\}$ and $\left\{\epsilon_{i}\right\}$ satisfy

$$
\epsilon_{n} \leq \rho_{0}+\sum_{i=0}^{n-1} q_{i}+\sum_{i=0}^{n-1} k_{i} \epsilon_{i}, n \geq 1
$$

with $\rho_{0} \geq 0, q_{i} \geq 0, i \geq 0$. Then

$$
\epsilon_{n} \leq\left(\rho_{0}+\sum_{i=0}^{n-1} q_{i}\right) \mathrm{e}^{\sum_{i=0}^{n-1} k_{i}}, n \geq 1 .
$$

Existing studies show that we cannot compute collocation boundary value solutions by recurrences. All numerical values should be computed simultaneously through solving linear systems. Note that the element of $h \mathbf{A}(1: N, 2: N+1)$ is bounded by

$$
h\left(k_{1}+k_{2}+1\right) \bar{K}\left\|\sum_{i=-k_{1}}^{k_{2}+1} \phi_{i}^{k_{1}, k_{2}}(t)\right\|_{\infty} \leq h \bar{K} 2^{k_{1}+k_{2}+4},
$$

where $\bar{K}$ denotes the maximum of the kernel function $K(t, s)$, and the above inequality is derived from the Lesbegue constant of the polynomial interpolant (see [25]). We obtain $h \mathbf{A}(1: N, 2: N+1)<1$ whenever $h<\left(\bar{K} 2^{k_{1}+k_{2}+4}\right)^{-1}$, which enables us to compute $\operatorname{det}(\mathbf{I}-h \mathbf{A}(1: N, 2: N+1)) \neq 0$ by Gaussian elimination, as is done in [22]. Therefore, the well-posedess of the solution computed by $G M C_{k_{1}, k_{2}} M$ is guaranteed. It is noted that when we encounter stiff problems, the maximum $\bar{K}$ may be 
large, which implies we have to apply a particularly small stepsize $h$ and restricts the application of the collocation method. However, due to the compactness of Volterra integral operator, the spectrum of $h \mathbf{A}(1: N, 2: N+1)$ will be found in the neighborhood of 0 with a tolerance stepsize, and the multistep collocation method is feasible in practical uses. In Figure 1, we show the discretized spectrum of $h \mathbf{A}(1: N, 2: N+1)$ by considering the kernel function $K(t, s)=50 \mathrm{e}^{\mathrm{i} \omega(t-s)}$ with the maximum 50 . It can be seen that eigenvalues are bounded by the unit circle when the stepsize decreases to 1/64, which guarantees the solvability of the linear system.
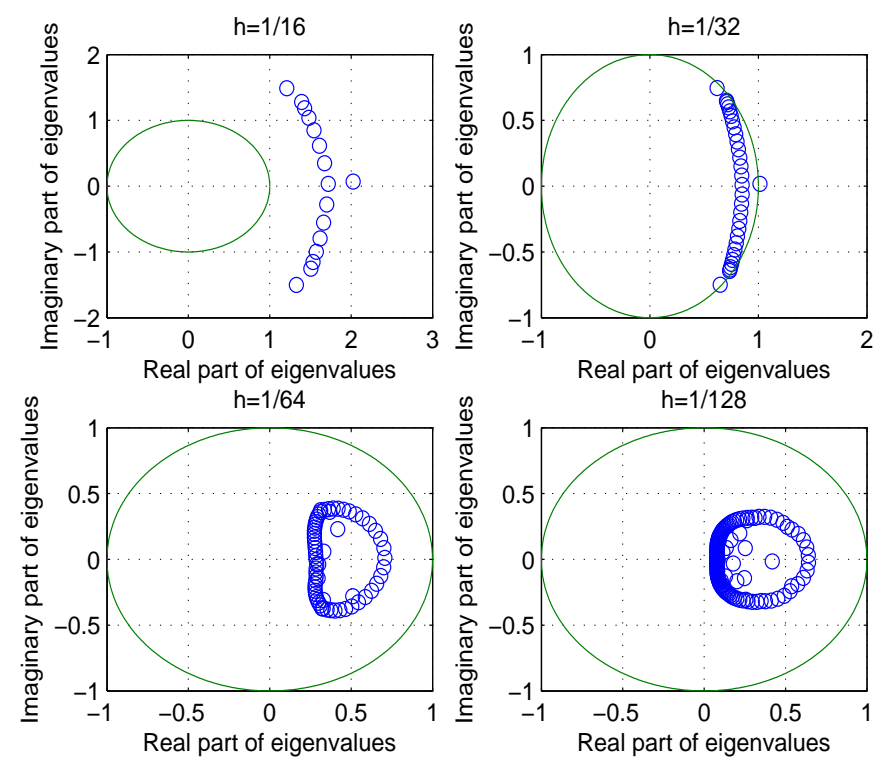

Figure 1. The spectrum of $h \mathbf{A}(1: N, 2: N+1)$ for various stepsizes $h$.

Furthermore, we arrive at the following theorem by employing Lemmas 1 and 2.

Theorem 3. Suppose that $K(t, s), f(t)$ in VIE (1) are sufficiently smooth, that is, $K \in C^{k_{1}+k_{2}+2}(D)$ and $f \in C^{k_{1}+k_{2}+2}(I)$. Furthermore, let $u_{h}(t)$ denote the collocation polynomial computed by $G M C_{k_{1}, k_{2}} M$ with a stepsize $h$. Then the collocation error $e_{h}(t)=u(t)-u_{h}(t)$ in the collocation grid is bounded by

$$
\max _{t \in X_{h}}|e(t)| \leq C h^{k_{1}+k_{2}+2},
$$

where the constant $C$ is independent of the stepsize $h$ but depends on $T$.

Proof. Note that by

$$
u(t)=f(t)+\int_{0}^{t} K(t, s) u(s) d s, t \in[0, T]
$$

and

$$
u_{h}(t)=f(t)+\int_{0}^{t} K(t, s) u_{h}(s) d s, t \in X_{h}
$$

we obtain the collocation error function $e_{h}(t)$ satisfying

$$
e_{h}(t)=\int_{0}^{t} K(t, s) e_{h}(s) d s, t \in X_{h} .
$$

Let

$$
R_{n}^{k_{1}, k_{2}}(v):=\int_{0}^{k_{1}+k_{2}+1} \kappa_{k_{1}}^{k_{2}}(v, z) u^{\left(k_{1}+k_{2}+2\right)}\left(t_{n}+z h\right) d z
$$


where

$$
\kappa_{k_{1}}^{k_{2}}(v, z):=\frac{1}{\left(k_{1}+k_{2}+1\right) !}\left((v-z)_{+}^{k_{1}+k_{2}+1}-\sum_{j=-k_{1}}^{k_{2}+1} \phi_{j}^{k_{1}, k_{2}}(v)(j-z)_{+}\right) .
$$

For $t \in\left[0, t_{k_{1}}\right]$, we have

$$
e_{h}(t)=e_{h}\left(t_{k_{1}}+s h\right)=\sum_{i=-k_{1}}^{k_{2}+1} v_{k_{1}+i} \phi_{i}^{k_{1}, k_{2}}(s)+h^{k_{1}+k_{2}+2} R_{0}^{k_{1}, k_{2}}(s), s \in\left[-k_{1}, 0\right],
$$

where $v_{n}:=e_{h}\left(t_{n}\right)$. For $t \in\left[t_{j}, t_{j+1}\right], j=k_{1}, \cdots, N-k_{2}-1$, we have

$$
e_{h}(t)=e_{h}\left(t_{j}+s h\right)=\sum_{i=-k_{1}}^{k_{2}+1} v_{j+i} \phi_{i}^{k_{1}, k_{2}}(s)+h^{k_{1}+k_{2}+2} R_{j}^{k_{1}, k_{2}}(s), s \in[0,1] .
$$

For $t \in\left[t_{N-k_{2}}, t_{N}\right]$, we have

$$
e_{h}(t)=e_{h}\left(t_{N-k_{2}-1}+s h\right)=\sum_{i=-k_{1}}^{k_{2}+1} v_{N-k_{2}+i} \phi_{i}^{k_{1}, k_{2}}(s)+h^{k_{1}+k_{2}+2} R_{N-k_{2}}^{k_{1}, k_{2}}(s), s \in\left[1, k_{2}+1\right]
$$

Furthermore, by letting

$$
\operatorname{RES}_{a, c, i}^{b, d}=\int_{a}^{b} K\left(t_{c}, t_{d}+s h\right) R_{i}^{k_{1}, k_{2}}(s) d s,
$$

we obtain

$$
v_{n}:= \begin{cases}h \sum_{i=-k_{1}}^{k_{2}+1} v_{i+k_{1}} \operatorname{MOM}_{k_{1}, n, 0}^{n-k_{1}, k_{1}}+h^{k_{1}+k_{2}+3} \operatorname{RES}_{k_{1}, n, 0}^{n-k_{1}, k_{1}}, & n=1, \cdots, k_{1}, \\ h \sum_{i=-k_{1}}^{k_{2}+1} v_{i+k_{1}} \mathrm{MOM}_{-k_{1}, n, i}^{0, k_{1}}+h^{k_{1}+k_{2}+3} \operatorname{RES}_{-k_{1}, n, 0}^{0, k_{1}} & \\ +h \sum_{l=k_{1}}^{n-1} \sum_{i=-k_{1}}^{k_{2}+1} v_{l+i} \mathrm{MOM}_{0, n, i}^{1, l}+h^{k_{1}+k_{2}+3} \sum_{l=k_{1}}^{n-1} \operatorname{RES}_{0, n, n-1}^{1, l} & n=k_{1}+1, \cdots, N-k_{2}, \\ h \sum_{i=-k_{1}}^{k_{2}+1} v_{i+k_{1}} \mathrm{MOM}_{-k_{1}, n, i}^{0, k_{1}}+h^{k_{1}+k_{2}+3} \operatorname{RES}_{0,0,0}^{k_{1}, 0} & \\ +h \sum_{l=k_{1}}^{N-k_{2}-1} \sum_{i=-k_{1}}^{k_{2}+1} v_{l+i} \mathrm{MOM}_{0, n, i}^{1, l}+h^{k_{1}+k_{2}+3} \sum_{l=k_{1}}^{N-k_{2}-1} \operatorname{RES}_{0, n, l}^{1, l} & \\ +h \sum_{i=-k_{1}}^{k_{2}+1} v_{i+N-k_{2}} \mathrm{MOM}_{1, n, i}^{n-N+k_{2}+1, n-k_{2}-1} & n=N-k_{2}+1, \cdots, N . \\ +h^{k_{1}+k_{2}+3} \operatorname{RES}_{1, n, N-k_{2}-1}^{n-N+k_{2}+1, N-k_{2}-1} & \end{cases}
$$


Suppose that $\operatorname{MOM}_{a, c, i}^{b, d}$ and $\operatorname{RES}_{a, c, i}^{b, d}$ are bounded by the constant $B$. It is easily noted from Equations (8) and (13) that $B$ does not depend on the stepsize. A direct calculation leads to

$$
\left|v_{n}\right| \leq \begin{cases}h B \sum_{i=-k_{1}, i \neq n-k_{1}}^{k_{2}+1}\left|v_{i+k_{1}}\right|+h\left|v_{n}\right|+h^{k_{1}+k_{2}+3} B, & n=1, \cdots, k_{1}, \\ h B \sum_{i=-k_{1}, i \neq n-k_{1}}^{k_{2}+1}\left|v_{i+k_{1}}\right|+h^{k_{1}+k_{2}+3} B+h\left(k_{1}+k_{2}+2\right)\left|v_{n}\right| & \\ +h B \sum_{l=k_{1}}^{n-1}\left(\sum_{i=-k_{1}, i \neq n-l}^{k_{2}+1}\left|v_{l+i}\right|+h^{k_{1}+k_{2}+3} B\right), & n=k_{1}+1, \cdots, N-k_{2}, \\ h B \sum_{i=-k_{1}, i \neq n-k_{1}}^{k_{2}+1}\left|v_{i+k_{1}}\right|+h^{k_{1}+k_{2}+3} B+h\left(k_{1}+k_{2}+2\right)\left|v_{n}\right| & \\ +h B \sum_{l=k_{1}}^{N-k_{2}-1}\left(\sum_{i=-k_{1}, i \neq n-l}^{k_{2}+1}\left|v_{l+i}\right|+h^{k_{1}+k_{2}+3} B\right) & \\ +h B \sum_{i=-k_{1}, i \neq n-N+k_{2}+1}^{k_{2}+1}\left|v_{i+N-k_{2}}\right|+h\left|v_{n}\right|+h^{k_{1}+k_{2}+3} B, & n=N-k_{2}+1, \cdots, N .\end{cases}
$$

Hence, we have

$$
\left(1-h\left(k_{1}+k_{2}+2\right)\right)\left|v_{n}\right| \leq h^{k_{1}+k_{2}+2} B+h\left(k_{1}+k_{2}+2\right) B \sum_{i=n+1}^{n+k_{2}}\left|v_{i}\right|+h\left(k_{1}+k_{2}+2\right) B \sum_{i=1}^{n-1}\left|v_{i}\right| .
$$

Since $1-h\left(k_{1}+k_{2}+2\right) \approx 1$ for sufficiently small stepsize $h$, we obtain

$$
\left|v_{n}\right| \leq h^{k_{1}+k_{2}+2} \tilde{B}+h k_{2}\left(k_{1}+k_{2}+2\right) \tilde{B}\left\|\mathbf{e}_{h}\right\|_{\infty}+h\left(k_{1}+k_{2}+2\right) \tilde{B} \sum_{i=1}^{n-1}\left|v_{i}\right| .
$$

According to Lemma 2, we have

$$
\left\|\mathbf{e}_{h}\right\|_{\infty} \leq \mathrm{e}^{\left(k_{1}+k_{2}+2\right) \tilde{B}} \tilde{B} h^{k_{1}+k_{2}+2}+h \mathrm{e}^{\left(k_{1}+k_{2}+2\right) \tilde{B}}\left(k_{1}+k_{2}+2\right) k_{2} \tilde{B}\left\|\mathbf{e}_{h}\right\|_{\infty},
$$

or equivalently,

$$
\left\|\mathbf{e}_{h}\right\|_{\infty} \leq \frac{\mathrm{e}^{\left(k_{1}+k_{2}+2\right) \tilde{B} \tilde{B}}}{1-h \mathrm{e}^{\left(k_{1}+k_{2}+2\right) \tilde{B}}\left(k_{1}+k_{2}+2\right) k_{2} \tilde{B}} h^{k_{1}+k_{2}+2}
$$

for sufficiently small stepsize $h$.

Example 1. Let us solve VIE with $G M C_{k_{1}, k_{2}} M$

$$
u(t)=\mathrm{e}^{t}+\int_{0}^{t} 2 \cos (t-s) u(s) d s, t \in[0,2]
$$

with the exact solution $u(t)=(1+t)^{2} \mathrm{e}^{t}$.

In this example, we test the performance of $G M C_{k_{1}, k_{2}} M$. We mainly focus on two terms of data, the maximum of error functions (INAE), and the convergence order. Computed results are shown in Tables 1-3. 
Table 1. Collocation error and convergence order of $G M C_{k_{1}, k_{2}} M$ for Example 1.

\begin{tabular}{lllll}
\hline & $\begin{array}{l}\mathbf{G M C}_{\mathbf{1 , 2}} \mathbf{M} \\
\text { Error }\end{array}$ & Order & $\begin{array}{l}\mathbf{G M C}_{\mathbf{1 , 3}} \mathbf{M} \\
\text { Error }\end{array}$ & Order \\
\hline$N=8$ & $4.10 \times 10^{-3}$ & - & $1.20 \times 10^{-3}$ & - \\
$N=16$ & $4.13 \times 10^{-4}$ & 3.31 & $2.72 \times 10^{-5}$ & 5.46 \\
$N=32$ & $1.59 \times 10^{-5}$ & 4.70 & $6.76 \times 10^{-7}$ & 5.33 \\
$N=64$ & $5.34 \times 10^{-7}$ & 4.90 & $1.22 \times 10^{-8}$ & 5.79 \\
$N=128$ & $1.72 \times 10^{-8}$ & 4.96 & $2.03 \times 10^{-10}$ & 5.91 \\
$N=256$ & $5.45 \times 10^{-10}$ & 4.98 & $3.27 \times 10^{-12}$ & 5.96 \\
Referenced Order & & 5.00 & & 6.00 \\
\hline
\end{tabular}

Table 2. Collocation error and convergence order of $G M C_{k_{1}, k_{2}} M$ for Example 1.

\begin{tabular}{lllll}
\hline & $\begin{array}{l}\mathbf{G M C}_{\mathbf{2 , 1}} \mathbf{M} \\
\text { Error }\end{array}$ & Order & $\begin{array}{l}\mathbf{G M C}_{2,3} \mathbf{M} \\
\text { Error }\end{array}$ & Order \\
\hline$N=8$ & $1.57 \times 10^{-2}$ & - & $9.39 \times 10^{-4}$ & - \\
$N=16$ & $4.77 \times 10^{-4}$ & 5.04 & $3.91 \times 10^{-6}$ & 7.91 \\
$N=32$ & $1.61 \times 10^{-5}$ & 4.89 & $2.62 \times 10^{-8}$ & 7.22 \\
$N=64$ & $5.32 \times 10^{-7}$ & 4.92 & $1.97 \times 10^{-10}$ & 7.05 \\
$N=128$ & $1.71 \times 10^{-8}$ & 4.96 & $1.51 \times 10^{-12}$ & 7.03 \\
Referenced Order & & 5.00 & & 7.00 \\
\hline
\end{tabular}

Table 3. Collocation error and convergence order of $G M C_{k_{1}, k_{2}} M$ for Example 1.

\begin{tabular}{lllll}
\hline & $\begin{array}{l}\mathbf{G M C}_{3, \mathbf{1}} \mathbf{M} \\
\text { Error }\end{array}$ & Order & $\begin{array}{l}\mathbf{G M C}_{\mathbf{3 , 2}} \mathbf{M} \\
\text { Error }\end{array}$ & Order \\
\hline$N=16$ & $1.58 \times 10^{-5}$ & - & $4.57 \times 10^{-7}$ & - \\
$N=32$ & $5.54 \times 10^{-7}$ & 4.83 & $1.51 \times 10^{-8}$ & 4.92 \\
$N=64$ & $1.12 \times 10^{-8}$ & 5.63 & $1.60 \times 10^{-10}$ & 6.57 \\
$N=128$ & $1.95 \times 10^{-10}$ & 5.85 & $1.38 \times 10^{-12}$ & 6.86 \\
Referenced Order & & 6.00 & & 7.00 \\
\hline
\end{tabular}

It can be seen from these tables that as the quantity of nodes increases, absolute errors decay fast, and as $k_{1}$ and $k_{2}$ get bigger, the convergence order enlarges. Besides, numerical results illustrate that $G M C_{k_{1}, k_{2}} M$ achieves the expected order of the estimate given in Theorem 3.

Remark 1. When numerical solutions of evolution equations are considered, Courant proposes that the combination of a consistent and stable numerical approach led to its convergence, which contributes to the foundation of classical numerical analysis theory of numerical studies on differential equations. On the other hand, the above convergence analysis is based on a fixed integration interval $[0, T]$, which differs from the convergence analysis for evolution problems where we usually consider the case of $T \rightarrow \infty$. In addition, it should be noted that the convergence result in Theorem 3 does not guarantee a feasible approximation in practical computation for long-time integration, especially when we are met with stiff problems. Therefore, we give linear stability analysis of the presented collocation method in the forthcoming subsection.

\subsection{Linear Stability Analysis}

For a long-time integration problem, round-off errors may dramatically affect the numerical solution. In this subsection, we analyze the collocation solution's linear stability originating from 
the study of numerical solutions of ordinary differential equations, where one usually considers the test equation

$$
y^{\prime}(t)=\lambda y(t), \operatorname{Re}(\lambda)<0
$$

Particularly, Brugnano and Trigiante investigated multistep methods for solving differential problems with the above scalar equation in [19]. For the general linear multistep formula

$$
\sum_{j=0}^{k} \alpha_{j} y_{n+j}-h \lambda \sum_{j=0}^{k} \beta_{j} y_{n+j}=0,
$$

we can introduce two polynomials

$$
\rho(z)=\sum_{j=0}^{k} \alpha_{j} z^{j}, \sigma(z)=\sum_{j=0}^{k} \beta_{j} z^{j}
$$

and define the associated characteristic polynomial $\pi(z, q)=\rho(z)-q \sigma(z)$ with $q=h \lambda$. When $\pi(z, q)$ is a Schur polynomial for fixed $q$, the method is absolutely stable at $q$. For the moment the definition of the region of absolute stability is

$$
\mathbb{D}:=\{q \in \mathbb{C}: \pi(z, q) \text { is a Schur polynomial }\}
$$

If $\mathbb{C}^{-} \subseteq \mathbb{D}$, the method is said to be $A$-stable.

Since both of discretization of ODE and VIE result in difference equations, we can investigate the generalized multistep collocation method with the help of stability studies of ODE. Consider the following test equation:

$$
u(t)=1+\lambda \int_{0}^{t} u(s) d s, t \in[0, T], \operatorname{Re}(\lambda)<0 .
$$

We turn to study the linear stability of the collocation solution by investigating Equation (15). By applying $G M C_{k_{1}, k_{2}} M$ we have

$$
y_{j}=1+\lambda \int_{0}^{j h} u_{h}(s) d s, j=k_{1}+1, \ldots, N-k_{2} .
$$

Next, noting the difference between $y_{j}$ and $y_{j-1}$ in Equation (15) leads to

$$
y_{j}-y_{j-1}=h \lambda \sum_{i=-k_{1}}^{k_{2}+1} y_{j-1+i} \int_{0}^{1} \phi_{i}^{k}(s) d s, j=k_{1}+1, \ldots, N-k_{2} .
$$

Then the characteristic polynomial is defined by

$$
\pi^{k_{1}, k_{2}}(z, q)=z^{k_{1}+1}-z^{k_{1}}-q \sum_{i=0}^{k_{1}+k_{2}+1} z^{i} \int_{0}^{1} \phi_{i-k_{1}}^{k_{1}, k_{2}}(s) d s=\rho(z)-q \sigma(z) .
$$

Before investigating the linear stability region, we introduce some helpful definitions and theorems in the version of $G M C_{k_{1}, k_{2}} M$.

Definition 4 ([19]). For any complex number $q:=h \lambda$, if the collocation solution $u_{h}$ to Equation (15) computed by $G M C_{k_{1}, k_{2}} M$ goes to 0 as $T$ goes $\infty$ for fixed stepsize, then $G M C_{k_{1}, k_{2}} M$ is said to be absolutely stable at $q$.

Definition 5 ([19]). For any $z \in \mathbb{S}$, if $G M C_{k_{1}, k_{2}} M$ is absolutely stable at $z$, then the set $\mathbb{S}$ is said to be the linear stability region of $G M C_{k_{1}, k_{2}}$ M. Particularly, if the left part of the complex plane is contained in $\mathbb{S}$, then $G M C_{k_{1}, k_{2}} M$ is said to be $A$-stable. 
Theorem 6 ([19]). For any complex number q, if roots of Equation (18) satisfy

$$
\left|z_{1}^{k}\right| \leq \cdots \leq\left|z_{k_{1}}^{k}\right|<1<\left|z_{k_{1}+1}^{k}\right| \leq \cdots \leq\left|z_{k_{1}+k_{2}+1}^{k}\right|
$$

then $G M C_{k_{1}, k_{2}} M$ is stable at $q$.

By a direct calculation, we find that roots of $\pi^{k_{1}, k_{2}}(z, q)$ do not satisfy the condition given in Theorem 6 in the case of $k_{1}=k_{2}$. Hence, the region of stability cannot be shown. In Figures 2 and 3 , we list the boundary locus corresponding to various multistep collocation methods with $k_{1} \neq k_{2}$, where the boundary $\Gamma$ is defined by

$$
\Gamma:=\left\{z \in \mathbb{C}, z=\frac{\rho\left(e^{i \theta}\right)}{\sigma\left(e^{i \theta}\right)}, 0 \leq \theta<2 \pi\right\} .
$$

It can be seen that these trajectories are Jordan curves, which implies $\Gamma$ is the boundary of corresponding absolute stability region. The stability region in Figure 2 is the part outside the boundary curves, while that in Figure 3 is the inside part. Therefore, we can conclude that $G M C_{k_{1}, k_{2}} M$ has wide stability region in the case of $k_{2}>k_{1}$. In addition, the boundary trajectories of $G M C_{k 1, k 2} M$ and $G M C_{k 2, k 1} M$ are symmetric with respect to virtual axis.

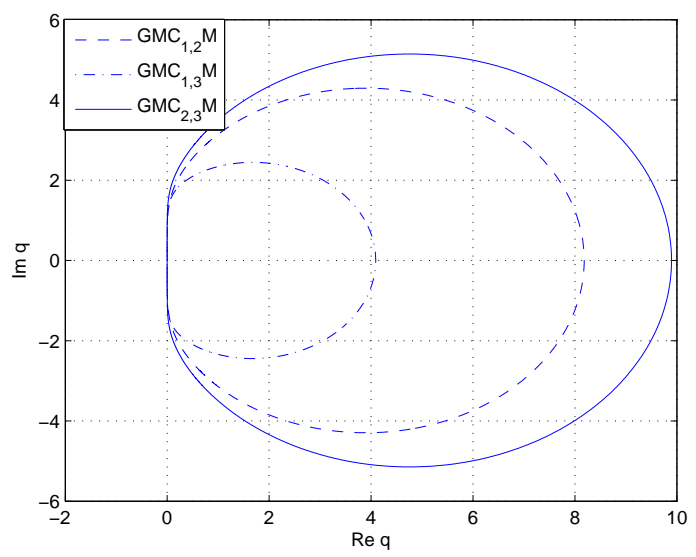

Figure 2. Linear stability region for $G M C_{1,2} M, G M C_{1,3} M, G M C_{2,3} M$.

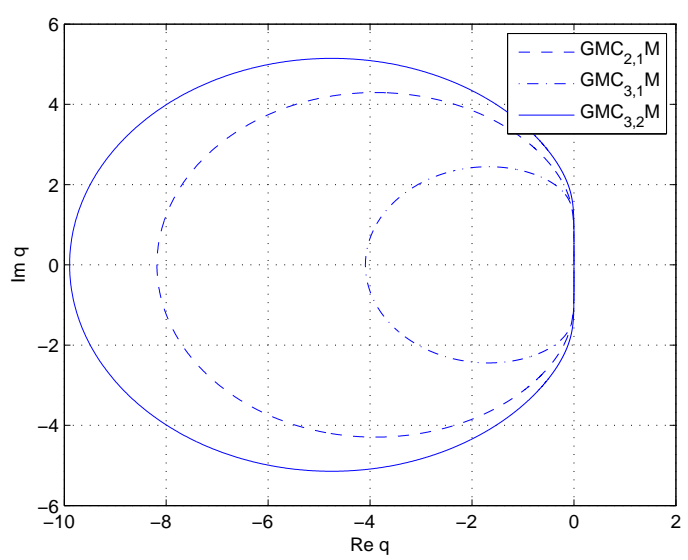

Figure 3. Linear stability region for $G M C_{2,1} M, G M C_{3,1} M, G M C_{3,2} M$. 
3. $\mathrm{GMC}_{\mathrm{k}_{1}, \mathrm{k}_{2}} \mathrm{M}$ in the Case of $\omega \gg 1$

When the oscillation parameter $\omega \gg 1$ in Equation (1), classical quadrature usually results in time-consuming algorithms. Hence, we first give an efficient numerical approach for moments in Equation (6) in this section. Then the frequency-explicit convergence analysis is presented.

\subsection{Fast Calculation of Moments}

Numerical studies of highly oscillatory integrals (HOIs) have been intensively focused on in the past few decades. High-order algorithms, such as Filon-type quadrature [26], Levin quadrature [27], and the numerical steepest decent method [28], have been proposed. In this subsection, we consider a composite quadrature rule based on Xiang's modified Filon-type quadrature developed in [29].

Consider the computation of

$$
\mathbf{M}_{\omega, n}^{a, b}:=\int_{a}^{b} K\left(t_{n}, s\right) \mathrm{e}^{\mathrm{i} \omega g\left(t_{n}, s\right)} \phi(s) d s, n=1,2, \cdots, N .
$$

When the phase has no stationary points, that is, $g^{\prime}\left(t_{n}, s\right) \neq 0$ for any $s \in[a, b]$, let $\left\{c_{k}\right\}_{k=0}^{v}$ be the equispaced nodes on the interval $[a, b]$, that is, $c_{k}=a+\frac{k}{v}(b-a)$ for $k=0, \cdots, v$. In addition, let $\left\{m_{k}\right\}_{k=0}^{v}$ denote a set of positive integers associated with nodes $\left\{c_{k}\right\}_{k=0}^{v}$, which helps represent Hermite interplant later. Furthermore, define the function

$$
\sigma_{k}(s)= \begin{cases}\frac{K\left(t_{n}, s\right) \phi(s)}{g^{\prime}\left(t_{n}, s\right)}, & k=1 \\ \frac{\sigma_{k-1}^{\prime}(s)}{g^{\prime}\left(t_{n}, s\right)}, & k \geq 2 .\end{cases}
$$

Then we can find a polynomial $p(s)=\sum_{q=0}^{\hat{N}} a_{q} s^{q}$ with $\hat{N}=\sum_{k=0}^{v} m_{k}-1$ satisfying

$$
\left\{\begin{array}{l}
p\left(g\left(c_{0}\right)\right)=\sigma_{1}\left(c_{0}\right) \\
\cdots \\
p^{\left(m_{0}-1\right)}\left(g\left(c_{0}\right)\right)=\sigma_{m_{0}-1}\left(c_{0}\right) \\
p\left(g\left(c_{1}\right)\right)=\sigma_{1}\left(c_{1}\right) \\
\cdots \\
p^{\left(m_{1}-1\right)}\left(g\left(c_{1}\right)\right)=\sigma_{m_{1}-1}\left(c_{1}\right) \\
\cdots \\
p\left(g\left(c_{v}\right)\right)=\sigma_{1}\left(c_{v}\right) \\
\cdots \\
p^{\left(m_{v}-1\right)}\left(g\left(c_{v}\right)\right)=\sigma_{m_{v}-1}\left(c_{v}\right)
\end{array}\right.
$$

With the coefficients $a_{q}$ by solving the above linear system, we can approximate $\mathrm{M}_{\omega, n}^{a, b}$ by

$$
\int_{g\left(t_{n}, a\right)}^{g\left(t_{n}, b\right)} p(s) \mathrm{e}^{\mathrm{i} \omega s} d s=\sum_{q=0}^{\hat{N}} a_{q} \int_{g\left(t_{n}, a\right)}^{g\left(t_{n}, b\right)} s^{q} \mathrm{e}^{\mathrm{i} \omega s} d s,
$$

where $\int_{g\left(t_{n}, a\right)}^{g\left(t_{n}, b\right)} s^{q} \mathrm{e}^{\mathrm{i} \omega s} d s$ can be calculated by incomplete Gamma function.

In the case of $g^{\prime}\left(t_{n}, s\right)=0$ for some $s \in[a, b]$, suppose $s=a$ without loss of generality. Then we insert the grid points

$$
a, a+\frac{2^{0}}{\omega}, a+\frac{2}{\omega}, a+\frac{2^{2}}{\omega}, \cdots, a+\frac{2^{m}}{\omega}, b,
$$


where $m$ is the maximum integer less than $\log _{2} \omega(b-a)$. Integration with Xiang's Filon quadrature in each subintervals results in the composite Filon quadrature. It is noted that the integral over the first interval is non-oscillatory and we can employ classical quadrature such as Gauss or Clenshaw-Curtis instead to avoid the stationary problem.

\subsection{Convergence Analysis with Respect to the Frequency}

Collocation methods with high-order quadrature usually lead to a class of fascinating algorithms, which are able to provide high-precision collocation solutions in the case of high frequency. In this subsection, we consider the general oscillator and investigate the convergence analysis for multistep collocation solutions, where the convergence order is represented by the frequency parameter $\omega$.

Firstly, let us restrict ourselves to considering the following set of functions.

Definition 7. Given any bivariate function $g(t, s)$ defined on $[0, T] \times[0, T]$, suppose that $g(t, s)$ has several stationary points $\xi_{1}, \cdots, \xi_{n_{t}}$ over $[0, T]$ for any fixed $t$, and

$$
\left\{\begin{array}{l}
g^{\prime}\left(t, \xi_{1}\right)=\cdots=g^{\left(r_{1}\right)}\left(t, \xi_{1}\right)=0, g^{\left(r_{1}+1\right)}\left(t, \xi_{1}\right) \neq 0 \\
g^{\prime}\left(t, \xi_{2}\right)=\cdots=g^{\left(r_{2}\right)}\left(t, \xi_{2}\right)=0, g^{\left(r_{2}+1\right)}\left(t, \xi_{2}\right) \neq 0, \\
\cdots \\
g^{\prime}\left(t, \xi_{n_{t}}\right)=\cdots=g^{\left(r_{N}\right)}\left(t, \xi_{n_{t}}\right)=0, g^{\left(r_{n_{t}}+1\right)}\left(t, \xi_{n_{t}}\right) \neq 0 .
\end{array}\right.
$$

Let $\rho(t)=\max _{i=1, \cdots, n_{t}}\left\{r_{i}\right\}$ and $r=\sup _{t \in[0, T]}\{\rho(t)\}$. Then $g(t, s)$ is said to be in $\mathcal{A}(r)$.

Secondly, we give a slight extension of the classical van der Corput Lemma (see [4] p. 333).

Lemma 8. Suppose that $g(t, s) \in \mathcal{A}(r)$. Moreover, suppose $\phi(s) \in C^{1}(a, b)$ and $\phi^{\prime}(s)$ is integrable. We can conclude that

$$
\left|\int_{a}^{b} \phi(s) \mathrm{e}^{\mathrm{i} \omega g\left(t_{n}, s\right)} d s\right| \leq C \omega^{-1 /(r+1)} \cdot n=1,2, \cdots, N .
$$

Here the constant $C$ is independent of $\omega$.

Finally, we are able to develop the convergence behavior of collocation polynomials computed by $G M C_{k_{1}, k_{2}} M$ in the highly oscillatory case.

Theorem 9. Assume both of $g(t, s) \in \mathcal{A}(r)$ and $f$ are sufficiently smooth. Then the numerical solutions derived from $G M C_{k_{1}, k_{2}} M$ for VIE (1) satisfy

$$
\max _{t \in I_{h}}\left\{\left|u(t)-u_{h}(t)\right|\right\}=O\left(\omega^{-1 /(r+1)}\right), \omega \rightarrow \infty .
$$

Proof. To begin with, we explore the boundedness of the solution $u(t)$ to Equation (1) and its derivative. By applying Picard iteration, we can rewrite $u(t)$ as

$$
u=f+\sum_{j=1}^{\infty}\left(\mathbf{K}^{j} f\right) .
$$

Here $\mathbf{K}$ denotes the integral operator

$$
(\mathbf{K} \phi)(t):=\int_{0}^{t} K(t, s) \mathrm{e}^{\mathrm{i} \omega g(t, s)} \phi(s) d s
$$


According to Lemma 8 , we get that $u(t)$ is bounded as $\omega \rightarrow \infty$. On the other hand, the derivative can be rewritten by a direct calculation

$$
\begin{aligned}
u^{\prime}(t) & =f^{\prime}(t)+\sum_{j=1}^{\infty}\left(K(t, t) f(t) \mathrm{e}^{\mathrm{i} \omega g(t, t)}\left(\mathbf{K}^{j-1} f\right)(t)+\mathrm{i} \omega \int_{0}^{t} K(t, s) f(s) g^{\prime}(t, s) \mathrm{e}^{\mathrm{i} \omega g(t, s)}\left(\mathbf{K}^{j-1} f\right)(s) d s\right) \\
& =f^{\prime}(t)+\sum_{j=1}^{\infty}\left(\mathcal{I}_{j}+\mathcal{I} \mathcal{I}_{j}\right),
\end{aligned}
$$

where

$$
\mathcal{I}_{j}:=K(t, t) f(t) \mathrm{e}^{\mathrm{i} \omega g(t, t)}\left(\mathbf{K}^{j-1} f\right)(t), \mathcal{I I}_{j}:=\mathrm{i} \omega \int_{0}^{t} K(t, s) f(s) g^{\prime}(t, s) \mathrm{e}^{\mathrm{i} \omega g(t, s)}\left(\mathbf{K}^{j-1} f\right)(s) d s .
$$

By letting $\omega \rightarrow \infty, \mathcal{I}_{j}$ is bounded due to Lemma 8 , and $\mathcal{I I}_{j}$ is bounded by noting that $g^{\prime}(t, s)$ vanishes at $s=0$.

When noting that the collocation error function $e_{h}(t)$ defined in the previous section satisfies

$$
e_{h}(t)=\int_{0}^{t} K(t, s) \mathrm{e}^{\mathrm{i} \omega g(t, s)} e_{h}(s) d s, t \in X_{h}
$$

we obtain

$$
(\mathbf{I}-h \mathbf{A}(1: N, 2: N+1)) \mathbf{e}_{h}=\mathbf{R},
$$

where

$$
\mathbf{e}_{h}=\left(\begin{array}{c}
e_{h}\left(t_{1}\right) \\
e_{h}\left(t_{2}\right) \\
\cdots \\
e_{h}\left(t_{N}\right)
\end{array}\right), \mathbf{R}=\left(\begin{array}{c}
h^{k_{1}+k_{2}+3} \operatorname{RES}_{0,0,0}^{1,0} \\
h^{k_{1}+k_{2}+3} \operatorname{REs}_{0,0,0}^{2,0} \\
\cdots \\
h^{k_{1}+k_{2}+3} \operatorname{REs}_{0,0,0}^{k_{1}, 0}+h^{k_{1}+k+2+3} \sum_{l=k_{1}}^{N-k_{2}-1} \operatorname{RES}_{0, N, N}^{1, l}+h^{k_{1}+k_{2}+3} \operatorname{REs}_{0, n, N-k_{2}-1}^{k_{2}+1, N-k_{2}-1}
\end{array}\right)
$$

Since both of $u(t)$ and $u_{h}(t)$ are bounded as $\omega \rightarrow \infty$, employing Lemma 8 implies

$$
\left|\mathrm{MOM}_{a, c, i}^{b, d}\right| \leq C \omega^{-1 /(r+1)},\left|\operatorname{RES}_{a, c, i}^{b, d}\right| \leq C \omega^{-1 /(r+1)} .
$$

Hence for fixed stepsize $h, \mathbf{I}-h \mathbf{A}(1: N, 2: N+1)$ is invertible for sufficiently large $\omega$, and we can represent $\mathbf{e}_{h}$ by

$$
\mathbf{e}_{h}=(\mathbf{I}-h \mathbf{A}(1: N, 2: N+1))^{-1} \mathbf{R} .
$$

By noting that maximum of $\mathbf{R}$ goes to 0 with a speed of $O\left(\omega^{-1 /(r+1)}\right)$ as $\omega$ goes to $\infty$, we obtain the estimate (21).

In the following example, we test the convergence rate of $G C M_{1,2} M$ in the case of high frequency.

Example 2. In this example, we solve the following VIE with $G C M_{1,2} M$,

$$
u(t)+\int_{0}^{t} e^{i \omega(t-s)} u(s) d s=e^{t}, t \in[0,1] .
$$

The exact solution is $u(t)=\left(\int_{0}^{t}\left(-c e^{s}\right) e^{-c s} d s+1\right) e^{c t}, c=i \omega-1$.

In Figure 4, we plot the scaled infinite norm of absolute error according to the corresponding order by letting $N=32$, and $\omega$ varies from 50 to 1000 . The left part shows the infinite norm of the error and the right part shows the absolute error scaled by corresponding rates. It can be seen that the 
increase of the frequency parameter $\omega$ makes the absolute error get smaller. This indicates as the kernel becomes more highly oscillatory, computed approximation becomes more accurate. Considering the right part of Figure 4, we find that when the frequency parameter $\omega$ reaches 150, the curve turns to a horizontal straight line, which is in agreement with the estimate given in Theorem 9.
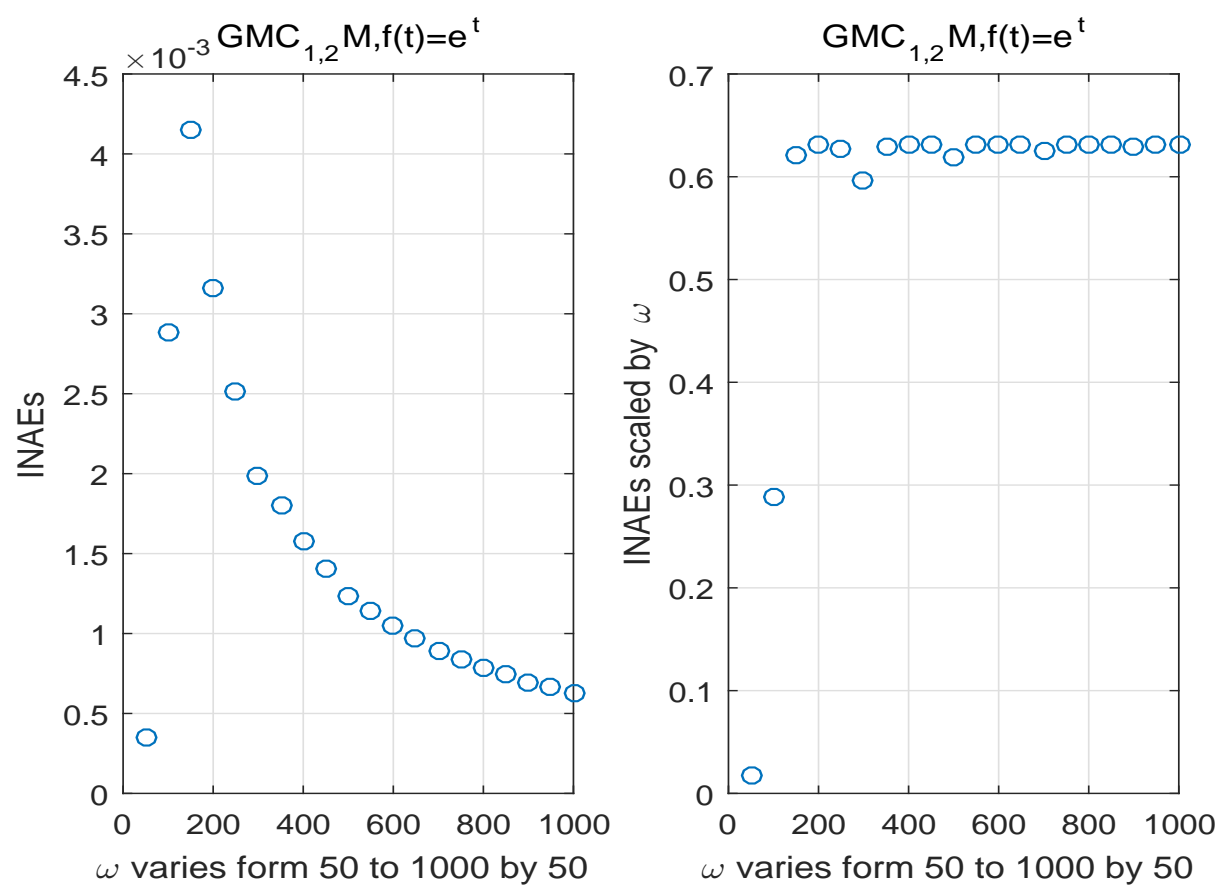

Figure 4. $G M C_{k_{1}, k_{2}} M$ for the highly oscillatory problem.

\section{Final Remark}

For VIEs with oscillatory and non-oscillatory kernels, we have investigated the generalized multistep collocation solution to VIE (1). Detailed convergence properties with respect to the stepsize and oscillation are presented. Noting that the new approach coupled with mild composite oscillatory quadrature rules is able to produce high-order approximation as the frequency goes to infinity, we could expect it is valuable to conduct further studies in related highly oscillatory problems, such as oscillatory Riemann-Hilbert problems, spectral calculation of oscillatory Fredholm operators, and so on.

Author Contributions: H.C. and J.M. conceived and designed the experiments; H.C. and L.L. performed the experiments; H.C. and J.M. analyzed the data; J.M.contributed reagents/materials/analysis tools; H.C. and J.M. wrote the paper. All authors have read and agreed to the published version of the manuscript.

Funding: This work was supported by National Natural Science Foundation of China (number 11901133) and the Science and Technology Foundation of Guizhou Province (number QKHJC[2020]1Y014).

Conflicts of Interest: The authors declare no conflict of interest.

\section{Abbreviations}

The following abbreviations are used in this manuscript:

VIE Volterra integral equation

$G M C_{k_{1}, k_{2}} M$ generalized multistep collocation method

ODE ordinary differential equation

CBVM collocation boundary value method

HOI highly oscillatory integral 


\section{References}

1. Brunner, H. Volterra Integral Equations: An Introduction to Theory and Applications; Cambridge University Press: Cambridge, UK, 2017.

2. Xiang, S.; Brunner, H. Efficient methods for Volterra integral equations with highly oscillatory Bessel kernels. BIT Numer. Math. 2013, 53, 241-263. [CrossRef]

3. Ma, J.; Xiang, S.; Kang, H. On the convergence rates of Filon methods for the solution of a Volterra integral equation with a highly oscillatory Bessel kernel. Appl. Math. 2013, 26, 699-705. [CrossRef]

4. E Stein. Harmonic Analysis: Real-Variable Methods, Orthogonality, and Oscillatory Integrals; Princeton University Press: Princeton, NJ, USA, 1993.

5. Xiang, S.; He, K. On the implementation of discontinuous Galerkin methods for Volterra integral equations with highly oscillatory Bessel kernels. Appl. Math. Comput. 2013, 219, 4884-4891. [CrossRef]

6. Ma, J.; Fang, C.; Xiang, S. Modified asymptotic orders of the direct Filon method for a class of Volterra integral equations. J. Comput. Appl. Math. 2015, 281, 120-125. [CrossRef]

7. Ma, J.; Kang, H. Frequency-explicit convergence analysis of collocation methods for highly oscillatory Volterra integral equations with weak singularities. Appl. Numer. Math. 2020, 151, 1-12. [CrossRef]

8. Fang, C.; He, G.; Xiang, S. Hermite-Type collocation methods to solve Volterra integral equations with highly oscillatory Bessel kernels. Symmetry 2019, 11, 168. [CrossRef]

9. Saira, S.X.; Liu, G. Numerical solution of the Cauchy-type singular integral equation with a highly oscillatory kernel function. Mathematics 2019, 7, 872. [CrossRef]

10. H. Brunner. Collocation Methods for Volterra Integral and Related Functional Equations; Cambridge University Press: Cambridge, UK, 2004.

11. Shen, J.; Tang, T.; Wang, L. Spectral Methods: Algorithms, Analysis and Applications; Springer: Berlin/Heidelberg, Germany, 2011.

12. Li, X.; Tang, T. Convergence analysis of Jacobi spectral collocation methods for Abel-Volterra integral equations of second kind. Front. Math. China 2012, 7, 69-84. [CrossRef]

13. Xie, Z.; Li, X.; Tang, T. Convergence analysis of spectral Galerkin methods for Volterra type integral equations. J. Sci. Comput. 2012, 53, 414-434. [CrossRef]

14. Cai, H.; Qi, J. A Legendre-Galerkin method for solving general Volterra functional integral equations. Numer. Algorithms 2016, 73, 1159-1180. [CrossRef]

15. Berrut, J.P.; Hosseini, S.A.; Klein, G. The linear barycentric rational quadrature method for Volterra integral equations. SIAM J. Sci. Comput. 2014, 36, A105-A123. [CrossRef]

16. Li, M.; Huang, C. The linear barycentric rational quadrature method for auto-convolution Volterra integral equations. J. Sci. Comput. 2019, 78, 549-564. [CrossRef]

17. Conte, D.; Paternoster, B. Multistep collocation methods for Volterra integral equations. Appl. Numer. Math. 2009, 59, 1721-1736. [CrossRef]

18. Fazeli, S.; Hojjati, G. Numerical solution of Volterra integro-differential equations by superimplicit multistep collocation methods. Numer. Algorithms 2015, 68, 741-768. [CrossRef]

19. Brugnano, L.; Trigiante, D. Solving Differential Problems by Multistep Initial and Boundary Value Methods; Cordon and Breach Science Publishers: Concord, ON, Canada, 1998.

20. Chen, H.; Zhang, C. Boundary value methods for Volterra integral and integro-differential equations. Appl. Math. Comput. 2011, 218, 2619-2630. [CrossRef]

21. Li, C.; Zhang, C. Block boundary value methods applied to functional differential equations with piecewise continuous arguments. Appl. Numer. Math. 2017, 115, 214-224. [CrossRef]

22. Ma, J.; Xiang, S. A collocation boundary value method for linear Volterra integral equations. J. Sci. Comput. 2017, 71, 1-20. [CrossRef]

23. Ma, J.; Liu, H. Fractional collocation boundary value methods for the second kind Volterra equations with weakly singular kernels. Numer. Algorithms 2020, 84, 743-760. [CrossRef]

24. Liu, L.; Ma, J. Block collocation boundary value solutions of the first-kind Volterra integral equations. Numer. Algorithms 2020, doi:10.1007/s11075-020-00917-6. [CrossRef]

25. Trefethen, L.N.; Weideman, J.A.C. Two results on polynomial interpolation in equally spaced points. J. Approx. Theory 1991, 65, 247-260. [CrossRef] 
26. Iserles, A.; Nørsett, S.P. Efficient quadrature of highly oscillatory integrals using derivatives. Proc. R. Soc. Math. Phys. Eng. Sci. 2005, 461, 1383-1399. [CrossRef]

27. Levin, D. Procedures for computing one- and two-dimensional integrals of functions with rapid irregular oscillations. Math. Comput. 1982, 38, 531-538. [CrossRef]

28. Milovanovic, G.V. Numerical calculation of integrals involving oscillatory and singular kernels and some applications of quadratures. Comput. Math. Appl. 1998, 36, 19-39. [CrossRef]

29. Xiang, S. Efficient Filon-type methods for $\int_{a}^{b} f(x) e^{i \omega g(x)} d x$. Numer. Math. 2007, 105, 633-658. [CrossRef]

Publisher's Note: MDPI stays neutral with regard to jurisdictional claims in published maps and institutional affiliations.

(C) 2020 by the authors. Licensee MDPI, Basel, Switzerland. This article is an open access article distributed under the terms and conditions of the Creative Commons Attribution (CC BY) license (http://creativecommons.org/licenses/by/4.0/). 